Revue scientifique francophone en Communication

organisationnelle

$18 \mid 2000$

Non-verbal, communication, organisation

\title{
La gestuelle d'accompagnement de la relation humain-ordinateur
}

\author{
Laurence Tobin
}

\section{OpenEdition}

Journals

Édition électronique

URL : https://journals.openedition.org/communicationorganisation/2429

DOI : 10.4000/communicationorganisation.2429

ISSN : 1775-3546

\section{Éditeur}

Presses universitaires de Bordeaux

\section{Édition imprimée}

Date de publication : 1 novembre 2000

ISSN : 1168-5549

Référence électronique

Laurence Tobin, "La gestuelle d'accompagnement de la relation humain-ordinateur », Communication et organisation [En ligne], 18 | 2000, mis en ligne le 27 mars 2012, consulté le 05 août 2021. URL: http://journals.openedition.org/communicationorganisation/2429; DOI : https://doi.org/10.4000/ communicationorganisation.2429

Ce document a été généré automatiquement le 5 août 2021 .

(C) Presses universitaires de Bordeaux 


\title{
La gestuelle d'accompagnement de la relation humain-ordinateur
}

\author{
Laurence Tobin
}

1 De quelle nature est la relation qui s'instaure entre une personne et son ordinateur? Cette relation est-elle simplement instrumentale et fonctionnelle ou déborde-t-elle sur les règles de l'interaction sociale? Peut-on parler de communication, de dialogue ? Une façon de tenter de répondre à ces questions est de se pencher sur la communication non-verbale, qui permet l'approche de processus mentaux et d'attitudes par ailleurs inobservables.

2 Des observations et des enregistrements vidéos «sur le vif» ont permis de dresser l'inventaire des gestes de l'interaction humain-ordinateur. Dans un deuxième temps, ces gestes observés chez les utilisateurs ont été classés dans cinq grands répertoires gestuels établis: synchronisation de l'interaction, expression corporelle d'affects, indication de contrôle social, contribution au plan du signifiant et gestes ergonomiques. Enfin, un modèle interprétatif, qui reste à tester, est proposé.

\section{La gestuelle dans la relation humain-ordinateur}

3 Le point de vue adopté ici n'est pas celui du geste ergonomique qui participe directement à l'interaction instrumentale avec l'ordinateur, mais des gestes qui accompagnent et ponctuent naturellement son utilisation. Les recherches bibliographiques faites dans divers domaines n'ont pas d'étude de ce type. Si la situation d'observation paraît nouvelle, le domaine de la communication non-verbale est lui bien établi. On a pris comme base descriptive les études classiques traitant des différents aspects de la gestuelle, parmi lesquelles celles de Jacques Cosnier, Marc-Alain Descamps, Jacques Corraze qui ont servi de source secondaire pour les résultats de chercheurs américains dont les textes restaient inaccessibles. Pour la gestuelle d'expression d'affects, on s'est appuyé sur le Non-verbal Dictionary en ligne de David Givens. 
4 En aval d'une meilleure connaissance des aspects émotionnels, relationnels et non verbaux de l'interaction humain - ordinateur se trouvent des enjeux importants, dont la recherche d'interfaces plus performantes. Au MIT par exemple, plusieurs équipe travaillent sur l'enrichissement des possibilités interactionnelles avec l'ordinateur. Les travaux de Justine Cassell sont intéressants. Ils portent sur la conception d'un interface - REA, automate doué de parole et de gestuelle co-verbale - qui incorpore les connaissances sur la communication non-verbale provenant d'observations de dialogues naturels. Ceci permet de tester les connaissances sur la gestuelle et de rectifier certaines conclusions, par exemple sur le rôle du regard dans le passage des tours de parole. L'organisation informationnelle du contenu de l'interaction en thèmerhème interviendrait également de manière décisive pour motiver les gestes d'emphase. Toujours au MIT. Citons également les travaux menés par l'équipe de Rosalind Picard qui portent sur les réactions émotionnelles liées à l'utilisation de nouvelles technologies et particulièrement au décryptage de ces réactions et leur prise en compte par des ordinateurs d'un nouveau genre. L'identification des émotions se fait à partir d'indicateurs physiologiques plutôt qu'à partir du comportement non-verbal.

5 D'autres travaux, portant sur des problèmes connexes, éclairent aussi notre problématique. The Media Equation, publié en 1996 par Byron Reeves et Clifford Nass, de Stanford University, fait état de nombreuses expériences comportementales tendant à démontrer que les utilisateurs étendent à l'ordinateur les règles de l'interaction sociale. L'ordinateur se voit attribuer une présence sociale à laquelle les utilisateurs réagissent comme à celle d'une autre personne. Reste à savoir si l'ordinateur constitue une exception ou une nouveauté, ou si tout face à face avec un artefact s'est de tout temps calqué sur le dialogue humain?

\section{Les observations}

6 Environ une douzaine d'heures d'observations ont été faites avec divers groupes étudiants de l'IUP Génie Informatique et Statistique de Vannes. Les étudiants travaillent seuls ou en binôme, et parfois en groupes plus grands. Des observations crayon/papier ont permis aux étudiants de s'habituer à être observés et ont été suivies de l'installation d'une caméra dans les salles de cours. Les plans sont en grande partie fixes, mais pour quelques séances, on a essayé de capter des moments particuliers, avec plus ou moins de bonheur.

7 D'autres enregistrements, environ 12 heures, ont été faits sur un universitaire, dans son bureau, en plan fixe avec cadrage permettant de capter toute la partie supérieure du corps et le plan de travail.

8 Quatre collègues enseignants d'informatique, interviewés pour cette étude, ne rapportent pas (ou peu) de cas d'emportement et d'agression verbale des étudiants envers leurs ordinateurs mais sont plusieurs à relever que les comportements diffèrent selon les phases de travail. La première phase correspond à la prise de connaissance du problème posé et est gestuellement relativement calme. La deuxième phase est celle de la réflexion, et des essais et échecs au cours desquels les étudiants semblent anxieux et agités. La troisième phase est la rédaction, la mise au point, et la documentation des tâches demandées. C'est une phase d'entrée de donnée, sans heurt particulier, mais très rapide. 
9 Toutes les observations ainsi réalisées ont été « opportunistes ». Elles se sont greffées sur des moments réels de la vie des étudiants. Ceci a permis d'observer des comportements « quasi » naturels mais il en découle un certain nombre de problèmes : des problèmes liés à la qualité médiocre des enregistrements - manque de lumière, manque de détails, impossibilité d'enregistrer l'ensemble des données pertinentes avec une seule caméra -, et des problèmes méthodologiques concernant le manque de contrôle et d'homogénéité des tâches effectuées par les étudiants.

Les conditions mêmes de l'étude en ont fixé les limites. C'est une recherche exploratoire destinée, à ce stade, à jalonner le domaine et générer des hypothèses.

\section{Les gestes observés}

11 Les utilisateurs sont loin d'être impassibles devant leur écran. Leur gestuelle est riche et diverse mais cependant moins dense que celle du dialogue humain. Les différents types de gestes concernés sont ici passés en revue rapidement et de façon impressionniste.

\section{Le regard}

12 Le regard est mobile. Il est dirigé de façon intermittente vers l'écran et une bonne partie du temps vers le clavier. Lorsque c'est le cas, il ne semble pas que ce soit toujours pour vérifier la frappe. À d'autres moments, le regard est dirigé sur des papiers qui font partie de la situation de travail. Une proportion non négligeable de ces regards se dirige vers un ailleurs sans objet précis, vers le haut ou vers le coté. C'est un regard intérieur correspondant à une phase de réflexion. Il faut enfin mentionner les regards d'évasion, dirigés vers la fenêtre ou la porte.

\section{Les mains}

Les mains sont très actives. Pour une partie du temps, elles gèrent le clavier et la souris. Mais elles s'évadent souvent, parfois de façon concomitante avec le regard. Lorsque les mains s'échappent, elles se rangent près des genoux, elles soutiennent la tête ou plus souvent, elles accomplissent des gestes nombreux et variés d'auto-contact: mainlèvres, main-menton, main-front, main-cou, main-nuque. Ces gestes grattent, touchent, pressent, caressent. Ils sont omniprésents, et il est possible qu'ils se propagent par contagion. Les gestes d'auto-contact sont plus nombreux dans la phase médiane de la tâche. On observe aussi des manipulations d'objets, dont certaines sont expertes et ritualisées (de type majorette avec un crayon). On observe également quelques gestes déictiques qui situent un point sur l'écran sans que ce geste s'adresse nécessairement à un interlocuteur. Les mains ont enfin à charge les gestes instrumentaux avec le clavier et la souris, qui sont accomplis avec plus ou moins de force et de grâce. Ces gestes instrumentaux sont parfois suspendus. La main reste sur la souris, inactive, ou les deux mains sont au-dessus du clavier ou en contact avec lui, sans bouger, prêtes à l'action. Plus rarement on observe des gestes de contact où les étudiants touchent et prennent à pleines mains le côté du clavier ou de l'écran. 


\section{Le visage}

14 La position des sourcils - neutres, relevés ou froncés -, et de la bouche - ouverte, froncée en bourse, tendue, lèvres rentrées vers l'intérieur-accompagnent le travail mental et jouent peut-être un rôle dans la synchronisation.

a respiration est difficile à capter sur les enregistrements. C'est dommage car les reprises de souffle, les expirations, les soupirs et bâillements jouent sans doute un rôle dans la gestuelle de synchronisation que l'on a relevée par ailleurs.

\section{Les changements de posture}

Les changements de posture sont fréquents et réguliers. On observe un mouvement de va-et-vient entre une position rapprochée de l'ordinateur, buste droit ou penché vers l'avant, jambes repliées (mais pas toujours) et une posture distanciée, ou la chaise est repoussée plus loin, le buste incliné vers l'arrière ou affalé et les jambes étendues (mais pas toujours). Les étudiants s'assoient, puis au bout d'un moment repoussent leur chaise pour adopter une attitude distanciée puis saisissent leur chaise des mains pour se rapprocher et ainsi de suite. Les positions de côté, formant un angle avec l'écran de l'ordinateur sont moins fréquentes dans notre corpus on trouve également des étirements de confort.

17 Pour récapituler ce catalogue de gestes en utilisant les classifications classiques du dialogue humain, on voit que certaines catégories de gestes sont notoirement absentes. On ne trouve qu'un seul geste métaphorique, celui des doigts, suspendus au-dessus du clavier, qui miment le geste de taper. Ce geste cependant se trouve chez plusieurs personnes. On ne trouve également pas d'emblématiques, le doigt levé pour appeler l'enseignant mis à part. Les déictiques sont peu fréquents mais se trouvent sporadiquement chez plusieurs usagers. En revanche, on trouve des gestes bâtons qui se greffent sur les gestes de frappe au clavier, des gestes de synchronisation, phatiques (regard) et régulateurs (toucher), et une grande abondance des gestes traditionnellement classés dans les extra-communicatifs : auto contacts, déplacements posturaux et gestes des mains.

Pour l'observateur, ce mouvement perpétuel est intriguant. Tout comme pour la gestuelle co-verbale, rien ne semble complètement livré au hasard, la chorégraphie semble coller à la situation et remplir une fonction, ne serait ce que pour celui qui l'exécute. Le modèle explicatif avancé ici ne l'est qu'à tire provisoire. Il devra être vérifié sur des corpus mieux étayés.

\section{La gestuelle ergonomique}

Hypothèse 1: La gestuelle ergonomique sert de support à d'autres fonctions et en particulier, elle sert de véhicule à la gestuelle de synchronisation.

20 Même si ce n'est pas elle qui nous intéresse au premier plan, la gestuelle fonctionnelle de l'interaction humain-ordinateur est bien présente dans nos corpus : maniement du clavier et de la souris regard posé sur l'écran. Pour le clavier, le geste sélectionne et active les touches pour encoder un message verbal ou numérique. Pour la souris qui substitue un déplacement horizontal sur le plan de travail au déplacement sur l'écran, 
le geste montre (deixis) et sélectionne. L'écran affiche les données qui ont été fournies (rétroaction « miroir »), ou l'exécution d'une commande (réponse) sous forme visuelle qui appelle le regard.

21 Sur cette gestuelle fonctionnelle "froide " vont se greffer des variations de style individuelles, des variations co-textuelles, et des effets d'interaction qui miment le face à face.

22 Pour ce qui est du maniement du clavier, on voit clairement se profiler des styles personnels très différents d'un usager à l'autre. La force et le rythme varient de l'affleurement des touches à une véritable percussion. On peut penser que les styles varient selon les personnalités, les attitudes, et les états émotionnels ${ }^{1}$.

Pour un même individu, les variations de rythme et de force seraient liées au contenu de l'interaction. Le martèlement du clavier, varierait avec la progression du texte tapé tout comme la chaîne sonore ralentit ou accélère pour accommoder et suivre le travail de conception verbale.

Le mouvement de haut en bas pour percuter les touches est similaire aux gestes «bâtons" qui marquent l'emphase. Si ces gestes d'emphase au clavier prennent les mêmes fonctions que ceux du dialogue, ils soulignent des unités de contenu et participent au déroulement de l'interaction. Ce serait le cas également pour les gestes "suspendus » que l'on observe au clavier et à la souris, qui serviraient au maintien du tour de parole. Le regard, enfin, qui sert à la lecture de l'écran, mimiquerait lui aussi le passage des tours de parole et participerait à la structuration thématique ${ }^{2}$. Ces points sont développés plus loin.

Un dernier point enfin, gestes au clavier et à la souris sont associés à la mise en route et à la phase d'arrêt de l'interaction homme machine : on connaît le geste de réveil de l'écran à l'aide de la souris qui consiste à la tapoter ou la faire glisser plusieurs fois. On peut penser à une poignée de main. La fonction d'appel est claire et sans doute renforcée par le bruit sur la surface du plan de travail. Notons qu'en situation de binôme - deux étudiants pour un ordinateur - clavier et souris sont souvent partagés, à tour de rôle, donnant lieu à un travail de coordination moteur aussi bien que cognitif.

\section{La gestuelle de synchronisation de l'interaction}

Hypothèse 2: Comme dans le monologue, la gestuelle de synchronisation de l'interaction assure sa structuration.

Dans le dialogue humain, les différents signaux émis par les interlocuteurs faisant savoir et valoir leurs intentions permettent que l'alternance des tours de parole se fasse sans heurts, sans chevauchements accidentels et sans perte de temps. Le monologue est une forme d'interaction apprise dont on a montré qu'elle était en partie calquée sur le dialogue ${ }^{3}$. Dans le monologue, les signes qu'un locuteur émet pour indiquer la macrostructure de son intervention (changement de thème par exemple) sont repris de la gestuelle de synchronisation du dialogue. Tout se passe comme si la même personne prenait tour à tour les rôles de locuteur et d'auditeur et se donnait la parole à elle même au cours d'un simulacre de passage de tour.

Certains indices semblent indiquer qu'un phénomène de même type soit à l'œuvre dans le face à face à l'ordinateur, au moins dans les tâches verbales. Cette macrostructuration de l'interaction ou du façonnage cognitif de la tâche met en scène le 
regard, les mains au clavier (en action et en attente), et le rythme et la force des mouvements au clavier.

Ainsi, les signaux de prise de parole seraient le détournement du regard de l'écran (regard détourné puis centration sur le clavier), l'inspiration ou le raclement de gorge, et l'adoption d'une posture rapprochée.

Des regards intermittents durant le tour de parole permettent la vérification de la bonne marche des opérations (rétroaction intra-tour) sans mettre en question le fait que c'est l'utilisateur qui a la parole. Ces regard de nature plus fugitive se caleraient à des moments de complétude grammaticale ou sémantique. Dans le dialogue humain la rétroaction permet au locuteur de vérifier que son message passe, qu'il est entendu et compris. Dans le face à face avec la machine, on vérifie que les données sont bien enregistrées et on contrôle leur qualité.

31 Pour maintenir son droit à la parole lors des pauses éventuellement prolongées, dues au processus de réflexion et d'élaboration cognitive, l'usager détournerait son regard, sur le coté ou sur le haut, ou même vers l'écran mais sans le regarder vraiment, tout en maintenant les mains étendues au dessus ou au contact du clavier. Quelquefois les doigts se délient en faisant semblant de taper dans un geste métaphorique. C'est un signal de suspension momentanée de l'interaction. Mais ce n'est pas la seule forme de pause que l'on observe. Dans d'autres cas, il y a bien détournement du regard mais accompagné de gestes d'auto-contact ou de détente.

Les signaux d'abandon de parole seraient la fin d'intervention au clavier parfois avec une force et un panache particuliers, le regard porté sur l'écran, l'expiration forcée (lèvres entrouvertes), l'adoption d'une posture distanciée: recul de la chaise, basculement du tronc en position arrière, ou éventuellement étirement des épaules vers l'arrière dans un geste de confort, le corps est aligné avec l'écran, les mains se replient hors du plan de travail, peut-être pour esquiver quelques gestes d'autocontacts ou se mettre au repos.

Ce premier ensemble de gestes est suivi de l'adoption de l'attitude plus en retrait de l'auditeur/spectateur. La main se saisit de la souris en permanence et lui imprime des mouvements minimaux pour éviter l'allumage de l'écran de veille. Ceci correspondrait aux interventions de régulation dans le dialogue (hochements de tête, oui, hm). L'émetteur se dédouble en récepteur pour évaluer sa propre production. Dans ce rôle les gestes d'auto-contact sont fréquents, peut-être pour pallier la passivité de la machine.

Les gestes d'emphase seraient concomitants du pilotage de l'interaction, dans le sens qu'ils n'apparaissent que pendant la prise de parole dans le dialogue et au clavier dans l'interaction humain-machine.

\section{La gestuelle d'expression d'affects}

Hypothèse 3 : Les affects exprimés concernent peu le rapport à la machine. La gestuelle expressive est surtout liée à l'effort de résolution de la tâche. Les gestes d'auto-contact et les déplacements posturaux aident également ce processus d'élaboration cognitive et ils suppléent l'impassibilité de l'ordinateur.

Dans le corpus recueilli, à quelques exceptions près, seules transparaissent sur les visages des usagers des mimiques qui trahissent l'effort de réflexion, sourcils froncés, 
bouche tendue, accompagnées de gestes autocentrés. Dans certains cas il est difficile de se rendre compte s'il s'agit de sentiments ressentis ou convoqués. Telle moue, dénote-telle un état dubitatif ou est-elle la représentation d'un raisonnement ou d'une rhétorique interne ? Si on suit la théorie de l'analyseur corporel ${ }^{4}$, on peut imaginer que cette gestuelle expressive soit en fait destinée à aider le processus d'élaboration cognitive. Par exemple, une mimique de contre-attaque servirait à aider à faire surgir un argument.

Les gestes d'auto-contact sont traditionnellement considérés comme la manifestation d'un état mental interne ne participant pas à la construction de l'interaction ou à sa signification. Effectivement, ils croissent lorsque les partenaires de la communication ne sont pas en contact direct, ce qui d'une façon est le cas ici ${ }^{5}$. Beaucoup de systèmes d'interprétation des gestes autocentrés construits pour des situations cliniques semblent hors propos et hors proportions pour les situations de travail qui nous intéressent. Par exemple, le geste qui consiste à se masser le cou, est en évolution directe du geste primitif d'esquive et de retrait et indique un désir de fuite, mais ne revêt-il pas d'autres fonctions dans les corpus qui nous concernent? Les gestes autocentrés seraient liés à une certaine anxiété ou frustration liées au labeur intellectuel. Lors d'une recherche intense et désordonnée, il y aurait un écho dans l'espace physique du brassage des éventualités et des solutions.

Ces gestes pourraient aussi avoir une fonction très similaire à celle que l'on vient de décrire pour les mimiques expressives, c'est à dire une fonction de stimulation de l'activité cognitive. Du fait que les terminaisons nerveuses des doigts et des lèvres sont extrêmement riches, la stimulation de ces zones permettrait de noyer les autres signaux et ainsi enrayer le stress dû à une surcharge informationnelle. Ces gestes d'auto-contact serviraient donc à se recentrer sur la tâche, tout en stimulant l'activité verbale localisée dans une zone proche au niveau du cerveau ${ }^{6}$.

\section{La gestuelle de contrôle social}

39 Hypothèse 4 : La gestuelle liée à la relation de soumission et de dominance qui pourrait nous permettre d'identifier un rapport particulier à l'objet technique, n'est que peu présente parce que l'interaction se construit d'abord sous la forme d'un dialogue de l'usager avec lui-même.

Peu de gestes donnent à voir un rapport de contrôle social dans la relation à l'ordinateur. Un élément gestuel pouvant éclairer ce rapport est le geste de préhension tactile sur le côté de l'écran ou du clavier qui indiquerait un rapport de dominance à l'objet. Un autre élément observé est la position debout pour taper sur le clavier de l'ordinateur dans un groupe d'étudiants « spécialistes » des réseaux, dans leur cours sur les réseaux.

41 On observe par ailleurs des personnalisations systématiques de l'ordinateur le panneau de configuration bien sûr, mais aussi l'extérieur affublé par quelques étudiants de personnages en plastique, posés en bas de l'écran, ou de leur casquette, posée sur le haut de l'écran. Dans les entretiens, certains enseignants indiquent que des étudiants donnent un nom à l'ordinateur (Marcel). Ce sont des tentatives de domestication 


\section{La gestuelle significative}

Les contributions au plan signifiant restent marginales. On a mentionné la correspondance possible entre la force avec laquelle le clavier est actionné et les gestes bâtons de l'oral. Dans ce cas on pourrait s'attendre à trouver ces gestes d'emphase dans les mêmes fonctions au moins dans les tâches verbales. Cette contribution au plan signifiant se ferait sous la forme de l'accentuation de l'élément Thématique de l'énoncé (les éléments informationnels nouveaux). Cassell indique également que les gestes bâtons interviennent pour les reprises d'erreurs, le discours rapporté et la contextualisation d'unités du discours. Autant d'hypothèses dérivées qu'il serait intéressant de tester avec des observations ad hoc.

\section{Conclusions}

Dialogue avec la machine ou dialogue avec soi-même ? L'utilisateur joue-t-il le rôle de la machine en sus du sien pour faciliter un échange limité ? Pour apporter des éléments de réponse probants, il faut quantifier à partir de la transcription minutieuse de corpus mieux construits.

Pour ce faire, il faut affiner chacune des hypothèses, mieux contrôler les observations, utiliser plusieurs caméras et surtout déterminer avec précision la tâche en cours, savoir s'il s'agit d'une tâche finalisée ou non (jeux, surf sur le web, travail), créative ou routinière, verbale ou numérique, ou enfin d'une tâche communicative (mail). Il faudrait en outre pouvoir suivre les progrès et les aléas de la tâche en cours ce qui est plus difficile. Il faudrait enfin pouvoir relier ces comportements avec ceux induits par l'utilisation d'autres artefacts, communiquant ou non, et avec des comportements de résolution de tâche sans ordinateur (examen par exemple).

Il n'est pas incongru de penser qu'un processus d'élaboration cognitive se fasse sur un mode dialogique. Si tel est le cas, nous devons dire à nos étudiants qu'il est normal, sinon souhaitable, qu'ils se parlent à eux-mêmes lorsqu'il travaillent. Nous devons encourager le travail en binômes. Nous pourrions aussi détecter les signaux nonverbaux des étudiants en difficulté. Au niveau des organisations cela relance l'importance du face à face et du groupe comme lieu d'actualisation de processus cognitifs. Il faudrait enfin se demander si le face à face prolongé avec l'écran d'un ordinateur à des conséquences sur le dialogue humain?

\section{BIBLIOGRAPHIE}

CASSELL J., «A Framework for Gesture Generation and Interprétation » In Computer Vision in Human-Machine Interaction, R. Cipolla and A. Pentland (eds.), Cambridge University Press. 2000.

CASSELL J., TORES 0., PRÉVOST S., « Turn Taking vs Discourse Analysis: How Best to Model Multimodal Conversation », in Y. Wilks (éd.), Machine Conversation, La Haye, Kluwer, 1999. 
CORRAZE J., Les Communications non-verbales, PUF, Le Psychologue. Paris, 1996.

COSNIER J., « Les Gestes du dialogue » in La Communication - État des savoirs, Paris, Éditions Sciences Humaines, 1998

COSNIER J., « Communication et Langages gestuels », in Les voies du langage, Dunod, 1982

COSNIER J., Brossard A., La Communication non-verbale, Neuchâtel, Delachaux et Niestlé, 1969.

DESCAMPS M-A., Le Langage du corps et la communication corporelle, PUF, Psychologie aujourd'hui, 1993.

GIVENS D., Non-verbal Dictionan; Center for Non-verbal Studies http://members.aol.com/ nonverbal2

PICARD R., Affective Computing, Massachusetts Institute of Technology, Media Laboratory Technical Report $\mathrm{N}^{\circ} 321,1995$.

REEVES B. \& NASS C, The Media Equation, Cambridge University Press, 1996.

TOBIN L., "Script et variations dans la parole médiatisée », Cahiers de l'Institut de Linguistique de Louvain, $\mathrm{n}^{\circ} 22,1996$, vol 1-2.

\section{NOTES}

1. Rosalind Picard, Affective Computing, MIT Media lab Technical Report $n^{\circ} 321,1995$.

2. Justine Cassell et al., " Turn Taking vs Discourse Structure », in Machine Conversations, lu Haye, Kluwer, 1999.

3. Laurence Tobin, "Script et variations dans la parole médiatisée ", Cahiers de l'Institut de Linguistique de Louvain, $n^{\circ} 22,1996$, vol. 1-2.

4. Jacques Cosnier, « Les Gestes du dialogue » in La Communication - Etat des savoirs, Paris, Editions Sciences Humaines, 1998.

5. Jacques Cosnier, « Communication et Langages gestuels », in Les voies du langage, Dunod, 1982

6. David Givens, "self touch» et "homonculus », Nonverbal Dictionary, (enter for Nonverbal Studies,

\section{AUTEUR}

\section{LAURENCE TOBIN}

Université de Bretagne-Sud 